\title{
A Study of Caristi's Fixed Point Theorem on Normed Space and Its Applications
}

\author{
Md. Abdul Mannan ${ }^{*}{ }^{\mathbb{D}}$, Moqbul Hossain1, Halima Akter ${ }^{1}$, Samiran Mondal ${ }^{2}$ \\ ${ }^{1}$ Department of Mathematics, Uttara University, Dhaka, Bangladesh \\ ${ }^{2}$ Department of Mathematics, Jashore University of Science and Technology, Jashore, Bangladesh \\ Email: ^mannan.iu31@gmail.com, abdul160064@gmail.com, akhalimaju@gmail.com, samiranmondal12@gmail.com
}

How to cite this paper: Mannan, Md.A. Hossain, M., Akter, H. and Mondal, S. (2021) A Study of Caristi's Fixed Point Theorem on Normed Space and Its Applications. Advances in Pure Mathematics, 11, 169-179.

https://doi.org/10.4236/apm.2021.113012

Received: January 8, 2020

Accepted: March 12, 2021

Published: March 15, 2021

Copyright (C) 2021 by author(s) and Scientific Research Publishing Inc. This work is licensed under the Creative Commons Attribution International License (CC BY 4.0)

http://creativecommons.org/licenses/by/4.0/

\begin{abstract}
In this work, we will discuss Caristi's fixed point theorem for mapping results introduced in the setting of normed spaces. This work is a generalization of the classical Caristi's fixed point theorem. Also, Caristi's type of fixed points theorem was partial discussed in Reich, Mizoguchi and Takahashi's and Amini-Harandi's results, we developed ideas that many known fixed point theorems can easily be derived from the Caristi theorem.
\end{abstract}

\section{Keywords}

Norm, Uniformity, Mizoguchi and Takahashi's, Rich's Problem, Caristi's

Fixed Point Theorem, Strong and Weak Contraction, Semi-Continuous

\section{Introduction}

This work was motivated by some recent works on Caristi's fixed point theorem for mappings defined on metric spaces with a partial order or graph. It seems that the terminology of graph theory instead of partial ordering gives clearer pictures and yields generalized fixed point theorems. The Caristi fixed point theorem is known as one of the very interesting and useful generalizations of the Banach fixed point theorem for self-mappings on a complete normed space. The Caristi's fixed point theorem is a modification of the $\mathcal{\varepsilon}$-variational principle of Ekeland ([1] [2]), which is a crucial tool in nonlinear analysis like optimization, variational inequalities, differential equations, and control theory. Furthermore, in 1977, Western [3] proved that the conclusion of Caristi's theorem is equivalent to norm completeness. In the last decades, Caristi's fixed point theorem has been generalized and extended in several directions (i.e. [4] [5] and the related references therein). Here at present, we discussed Caristi's fixed point theorem 
in normed spaces where Caristi's fixed point theorem was in matric space [6].

\section{Preliminaries}

We will discuss some applications of Caristi's fixed point theorem in complete normed space.

Theorem-1 [7]

Let $(X,\|\|$,$) be a complete normed space, and let T: X \rightarrow X$ be a mapping such that for

$$
\|x-T x\| \leq \phi(x)-\phi(T x), \forall x \in X .
$$

where $\phi: X \rightarrow[0, \infty)$ is a lower semi-continuous mapping. The $T$ has at least a fixed point.

We denote by $N$ the set of positive integers and by $R$ the set of real numbers.

Let $(X,\|\|$,$) be a complete normed space.$

We denote by $C B(X)$ the family of all no-empty closed and bounded subsets of $X$.

A function $H: C B(X) \times C B(X) \rightarrow[0, \infty)$.

Defined by $H\|A-B\|=\max \left\{\sup _{x \in B}\|x-A\|, \sup _{x \in A}\|x-B\|\right\}$ is said to be Housdorff norm on $C B(X)$ induced by the norm \|\| on $X$.

A point $v$ in $X$ is a fixed point of a map $T$ if $v=T v$ (when $T: X \rightarrow X$ is a single-valued map), or $v \in T v$ (when $T: X \rightarrow C B(X)$ is a multi-valued map).

Let $(X,\|\|)$ be a complete normed space and a map $T: X \rightarrow X$. Suppose there exists a function $\phi:[0, \infty) \rightarrow[0, \infty)$ satisfying $\phi(0)=0, \phi(s)<s$ for $s>0$ and suppose that $\phi$ is right upper semi-continuous such that $\|T x-T y\| \leq \phi\|x-y\|, \forall x, y \in X$. Then $T$ has a unique fixed point.

Problem-1 [8] [9] [10]

Let $(X, \|)$ be a complete normed space and let $T: X \rightarrow C B(X)$ be a multi-valued mapping such that $H(T x-T y) \leq \mu(\|x-y\|)$.

For all $x, y \in X$, where $\mu: R^{+} \rightarrow R^{+}$is continuous and increasing map such that $\mu(t)<t, \forall t>0$. Does $T$ have a fixed point?

In these works, the authors consider additional conditions on the mapping $\mu$ to find a fixed point.

1) Daffer et al. assumed that $\mu: R^{+} \rightarrow R^{+}$

i) $\mu$ is upper right semi-continuous;

ii) $\mu(t)<t, \forall t>0$ and;

iii) $\mu(t) \leq t-a t^{b}$ where $a>0,1<b<2$ on some interval $[0, s], s>0$.

2) Jachymski assumed that $\mu: R^{+} \rightarrow R^{+}$

i) $\mu$ is super additive i.e. $\mu(x+y)>\mu(x)+\mu(y), \forall x, y \in R^{+}$and;

ii) $t \rightarrow t-\mu(t)$ is non-decreasing.

Problem-2 [11]

Let $(X,\|\|)$ be a complete normed space and let $T: X \rightarrow C B(X)$ be a mapping such that $H\|T x-T y\| \leq \eta(\|x-y\|)\|x-y\|$. 
For all $x, y \in X$, where $\eta:(0,+\infty) \rightarrow[0,1)$ is a mapping such that $\lim \sup _{r \rightarrow t^{+}} \eta(r)<1$.

For all $r \in(0,+\infty)$. Does $T$ have a fixed point?

Theorem-2 [12]

Let $(X,\|\|)$ be a complete normed space and let $T: X \rightarrow C B(X)$ be a mapping such that $H\|T x-T y\| \leq \eta(\|x-y\|)\|x-y\|$.

For all $x, y \in X$, where $\eta:(0,+\infty) \rightarrow[0,1)$ is a mapping such that $\lim \sup _{r \rightarrow t^{+}} \eta(r)<1$.

For all $r \in(0,+\infty)$. Then $T$ has a fixed point.

\section{Normed Spaces [13]}

A normed on $X$ is a real function $\|\bullet\|: X \rightarrow R$ defined on $X$ such that for any $x, y \in X$ and for all $\lambda \in K$.

i) $\|x\| \geq 0$. ii) $\|x\|=0$ if and only if $x=0$. iii) $\|\lambda x\|=|\lambda|\|x\|$.

iv) $\|x+y\| \leq\|x\|+\|y\|$ (Triangle inequality).

A norm on $X$ defines a metric $d$ on $X$ which is given by

$$
d(x, y)=\|x-y\| ; x, y \in X
$$

and is called the metric induced by the norm.

The normed space is denoted by $(X,\|\bullet\|)$ or simply by $X$.

\section{Further Extension}

In this section, many of the known fixed point results can be deduced from light version of Caristi's theorem in normed space.

\section{Theorem-3}

Let $(X,\|\|$,$) be a complete normed space, and let T: X \rightarrow X$ be a mapping such that for

$$
\|x-y\| \leq \phi(x, y)-\phi(T x, T y), \forall x, y \in X .
$$

where $\phi: X \times X \rightarrow[0, \infty)$ is lower semi-continuous with respect to the first variable.

Then $\mathrm{T}$ has a unique fixed point.

\section{Proof:}

For each $x \in X$, let $y=T x$ and $\psi(x)=\phi(x, T x)$. Then for each $x \in X$.

$$
\therefore\|x-T x\| \leq \psi(x)-\psi(T x)
$$

and $\psi$ is a lower semi-continuous mapping.

If $\mathrm{X}$ is a normed space and $T: X \rightarrow X$ is a mapping, then

$$
\therefore\|x-T x\| \leq \phi(x)-\phi(T x), \forall x \in X
$$

where $\phi: X \rightarrow[0, \infty)$ is a lower semi-continuous mapping? And Thas at least a fixed point.

Comparing Equations (1) and (2), then we get $\psi: X \rightarrow[0, \infty)$ is a lower semi-continuous mapping and $T$ has a fixed point. 
Let $u, v$ be two distinct fixed points for $T$.

Now we will prove that the uniqueness of the fixed point i.e. $u=V$

$$
\begin{aligned}
& \therefore\|u-v\| \leq \phi(u, v)-\phi(T u, T v)=\phi(u, v)-\phi(u, v)=0 \\
& \Rightarrow\|u-v\| \leq 0 \\
& \text { i.e. } u-v=0 \\
& \Rightarrow u=v .
\end{aligned}
$$

Hence $T$ has a unique fixed point.

\subsection{Banach Contraction Principle}

Theorem-4 [14]

Let $(X,\|\|$,$) be a complete normed space, and let T: X \rightarrow X$ be a mapping such that for some $\alpha \in[0,1),\|T x-T y\| \leq|\alpha|\|x-y\|, \forall x, y \in X$.

Then $T$ has a unique fixed point.

Proof: We define $\phi(x, y)=\frac{\|x-y\|}{1-|\alpha|}$. Then we have

$$
(1-|\alpha|)\|x-y\| \leq\|x-y\|-\|T x-T y\|
$$

It means that

$$
\begin{aligned}
& \|x-y\| \leq \frac{\|x-y\|}{1-|\alpha|}-\frac{\|x-y\|}{1-|\alpha|} \\
& \Rightarrow\|x-y\| \leq \phi(x, y)-\phi(T x, T y) \\
& \Rightarrow\|x-y\| \leq \phi(x, y)-\phi(x, y) \\
& \Rightarrow\|x-y\| \leq 0 \\
& \text { i.e. } x-y=0 \\
& \Rightarrow x=y .
\end{aligned}
$$

Hence $T$ has a unique fixed point.

\section{Theorem-5}

Let $(X,\|\|$,$) be a complete normed space, and let T: X \rightarrow X$ be a mapping such that

$$
\|T x-T y\| \leq|\eta|\|x-y\|, \forall x, y \in X
$$

where $\eta:[0,+\infty) \rightarrow[0, \infty)$ is lower semi-continuous mapping such that $\eta(t)<t$, for each $t>0$ and $\frac{\eta(t)}{t}$ is a non-decreasing map. Then $T$ has a unique fixed point.

Proof:

Define $\phi(x, y)=\frac{\|x-y\|}{1-\frac{|\eta|\|x-y\|}{\|x-y\|}}$ if $x \neq y$ and otherwise $\phi(x, x)=0$. Then we have

$$
\|T x-T y\| \leq|\eta|\|x-y\|
$$




$$
\begin{aligned}
& \Rightarrow-\|T x-T y\| \geq-|\eta|\|x-y\| \\
& \Rightarrow\|x-y\|-\|T x-T y\| \geq\|x-y\|-|\eta|\|x-y\| \\
& \Rightarrow\|x-y\|\left[1-\frac{|\eta|\|x-y\|]}{\|x-y\|}\right] \leq\|x-y\|-\|T x-T y\| \\
& \Rightarrow\|x-y\| \leq \frac{\|x-y\|-\|T x-T y\|}{1-\frac{|\eta|\|x-y\|}{\|x-y\|}} \\
& \Rightarrow\|x-y\| \leq \frac{\|x-y\|}{1-\frac{|\eta|\|x-y\|}{\|x-y\|}}-\frac{\|T x-T y\|}{1-\frac{|\eta|\|x-y\|}{\|x-y\|}}
\end{aligned}
$$

Since $\frac{|\eta(t)|}{t}$ is non-decreasing and $\|T x-T y\|<\|x-y\|$

$$
\begin{aligned}
& \therefore\|x-y\| \leq \frac{\|x-y\|}{1-\frac{|\eta|\|x-y\|}{\| x-y \mid}}-\frac{\|T x-T y\|}{1-\frac{|\eta|\|x-y\|}{\|x-y\|}}=\phi(x, y)-\phi(T x, T y) \\
& \Rightarrow\|x-y\| \leq \phi(x, y)-\phi(x, y) \\
& \Rightarrow\|x-y\| \leq 0 \\
& \therefore x-y=0 \\
& \Rightarrow x=y
\end{aligned}
$$

Hence Thas a unique fixed point.

\section{Theorem-6}

Let $(X,\|\|$,$) be a complete normed space, and let T: X \rightarrow C B(X)$ be a non-expansive mapping such that, for each $x \in X$, and for all $y \in T x$, there exists $z \in T y$ such that $\|x-y\| \leq \phi(x, y)-\phi(y, z)$ where $\phi: X \times X \rightarrow[0, \infty)$ is lower semi-continuous mapping w. r. to the first variable. Then $T$ has a fixed point.

\section{Proof:}

Let $x_{0} \in X$ and $x_{1} \in T x_{0}$. If $x_{0}=x_{1}$, then $x_{0}$ is a fixed.

Now we will prove that $T$ has a fixed point.

If $x_{0} \neq x_{1}, \exists x_{2} \in T x_{1}$ such that

$$
\left\|x_{0}-x_{1}\right\| \leq \phi\left(x_{0}, x_{1}\right)-\phi\left(x_{1}, x_{2}\right)
$$

Alternatively, we choose $x_{n} \in T x_{n-1}$ such that $x_{n}=x_{n-1}$ and we find $x_{n+1} \in T x_{n}$ such that

$$
0<\left\|x_{n-1}-x_{n}\right\| \leq \phi\left(x_{n-1}, x_{n}\right)-\phi\left(x_{n}, x_{n+1}\right)
$$

which means that $\left\{\phi\left(x_{n-1}, x_{n}\right)\right\}_{n}$ is a non-increasing sequence and bounded below. So it is converges to $r \geq 0$.

From Equation (4), then we get

$$
\left\|x_{n-1}-x_{n}\right\|>0
$$

Taking the limit in Equation (5), then we get,

$$
x_{n-1}-x_{n}=0 \Rightarrow x_{n-1}=x_{n}
$$


Also for all $m, n \in N$ with $m>n$

$$
\begin{gathered}
\left\|x_{n}-x_{m}\right\| \leq \sum_{i=n+1}^{m}\left\|x_{i-1}-x_{i}\right\| \leq \sum_{i=n+1}^{m}\left[\phi\left(x_{i-1}, x_{i}\right)-\phi\left(x_{i}, x_{i+1}\right)\right] \\
\Rightarrow\left\|x_{n}-x_{m}\right\| \leq \phi\left(x_{n}, x_{n+1}\right)-\phi\left(x_{m}, x_{m+1}\right)
\end{gathered}
$$

Taking the limit sup on both sides in Equation (7), then we get,

$$
\begin{aligned}
& \lim _{n \rightarrow \infty}\left\{\sup \left\|x_{n}-x_{m}\right\| \leq \phi\left(x_{n}, x_{n+1}\right)-\phi\left(x_{m}, x_{m+1}\right)\right\} \\
& \Rightarrow\left\{\sup _{n \rightarrow \infty}\left\|x_{n}-x_{m}\right\|: m>n=0\right\}
\end{aligned}
$$

It means that $\left\{x_{n}\right\}$ is a Cauchy sequence and so it converges to $x \in X$ Now we will prove that $x$ is a fixed point of $T$.

$$
\begin{aligned}
&\|x-T x\| \leq\left\|x-x_{n+1}\right\|+\left\|x_{n+1}-T x\right\| \\
&=\left\|x-x_{n+1}\right\|+\left\|T x_{n}-T x\right\| \\
& \leq\left\|x-x_{n+1}\right\|+|\lambda|\left\|x_{n}-x\right\| \quad[\operatorname{Using}(6)] \\
&=\left\|x-x_{n}\right\|+|\lambda|\left\|x_{n}-x\right\| \\
&\|x-T x\| \leq\left\|x-x_{n}\right\|+|\lambda|\left\|x_{n}-x\right\|
\end{aligned}
$$

Taking the limit on both sides in Equation (8), then we get ,

$$
\begin{aligned}
& x-T x=0+|\lambda| \cdot 0 \\
& \Rightarrow x-T x=0 \\
& \Rightarrow T x=x
\end{aligned}
$$

Hence $\mathrm{T}$ has a unique fixed point.

\section{Theorem-7}

Let $(X,\|\|$,$) be a complete normed space, and let T: X \rightarrow C B(X)$ be a multi-valued function such that

$$
H(T x, T y) \leq|\eta|\|x-y\|, \forall x, y \in X
$$

where $\eta:[0, \infty) \rightarrow[0, \infty)$ is a lower semi-continuous map such that $\eta(t)<t, \forall t \in(0,+\infty)$ and $\frac{\eta(t)}{t}$ is a non-decreasing. Then $\mathrm{T}$ has a fixed point.

\section{Proof:}

Let $x \in X$ and $y \in T x$. If $y=x$, then $T$ has a fixed and the proof is complete.

We suppose that $y \neq x$. Then we define

$$
\theta(t)=\frac{\eta(t)+t}{2}, \forall t \in(0,+\infty)
$$

Since

$$
H(T x, T y) \leq|\eta|\|x-y\|<|\theta|\|x-y\|<\|x-y\|
$$

Thus there exists $\varepsilon>0$ such that

$$
|\theta|\|x-y\| \leq H(T x, T y)+\varepsilon
$$


So there exists $z \in T y$ such that

$$
\begin{gathered}
\|y-z\| \leq H(T x, T y)+\varepsilon \quad[\text { Using (10)] } \\
=|\theta|\|x-y\| \quad[\text { Using (9)] } \\
<\|x-y\| \\
\|y-z\|<\|x-y\|
\end{gathered}
$$

Again, we suppose that $y \neq z$, then we have

$$
\begin{aligned}
& \|x-y\|-|\theta|\|x-y\| \leq\|x-y\|-\|y-z\| \\
& \Rightarrow\|x-y\|(1-|\theta|) \leq\|x-y\|-\|y-z\| \\
& \Rightarrow\|x-y\| \leq \frac{\|x-y\|-\|y-z\|}{1-|\theta|}
\end{aligned}
$$

[Since $\frac{\theta(t)}{t}$ is also a non-decreasing function and $\left.\|y-z\|<\|x-y\|\right]$.

Define $\phi(x, y)=\frac{\|x-y\|}{1-|\theta|}$ if $x \neq y$, otherwise 0 for all $x, y \in X$.

From Equation (3), then we get,

$$
\begin{aligned}
& \|x-y\| \leq \phi(x, y)-\phi(y, z) \\
& \Rightarrow\|x-y\| \leq \phi(x, y)-\phi(T x, T y) \\
& \Rightarrow\|x-y\| \leq \phi(x, y)-\phi(x, y) \quad[\therefore y \in T x \text { and } z \in T y] \\
& \Rightarrow x-y=0 \\
& \Rightarrow x=y
\end{aligned}
$$

Hence $T$ has a unique fixed point.

\subsection{Mizoguchi-Takahashi's Type}

\section{Theorem-8}

Let $(X,\|\|$,$) be a complete normed space, and let T: X \rightarrow C B(X)$ be a multi-valued mapping such that

$$
H(T x, T y) \leq|\eta|(|| x-y \|)\|x-y\|, \forall x, y \in X
$$

where $\eta:[0, \infty) \rightarrow[0,1)$ is a lower semi-continuous and non-decreasing mapping? Then $T$ has a fixed point.

\section{Proof:}

Let $x \in X$ and $y \in T x$. If $y=x$, then $T$ has a fixed and the proof is complete.

Let $\theta(t)=\eta(t) t, \theta(t)<t, \forall t \in R_{+}$and $\frac{\theta(t)}{t}=\eta(t)$ is a non-decreasing mapping. Since,

$$
(T x-T y) \leq|\eta|(\|x-y\|)\|x-y||=|\theta(t)|\| x-y\|<\| x-y \|
$$

Thus there exists $\varepsilon>0$ such that

$$
|\theta(t)|\|x-y\| \leq H(T x, T y)+\varepsilon
$$


So there exists $z \in T y$ such that

$$
\begin{gathered}
\|y-z\| \leq H(T x, T y)+\varepsilon \quad[\text { Using (13)] } \\
=|\theta(t)|\|x-y\| \quad[\text { Using (12)] } \\
<\|x-y\| \\
\|y-z\|<\|x-y\|
\end{gathered}
$$

Again, we suppose that $y \neq z$, then we have

$$
\begin{aligned}
& \|x-y\|-\mid \theta(t)\|x-y\| \leq\|x-y\|-\|y-z\| \\
& \Rightarrow\|x-y\|(1-|\theta(t)|) \leq\|x-y\|-\|y-z\| \\
& \Rightarrow\|x-y\| \leq \frac{\|x-y\|-\|y-z\|}{1-|\theta(t)|}
\end{aligned}
$$

[Since $\frac{\theta(t)}{t}$ is also a non-decreasing function and $\|y-z\|<\|x-y\|$ ].

Define $\phi(x, y)=\frac{\|x-y\|}{1-|\theta(t)|}$ if $x \neq y$, otherwise 0 for all $x, y \in X$.

From Equation (15), then we get,

$$
\begin{aligned}
& \|x-y\| \leq \phi(x, y)-\phi(y, z) \\
& \Rightarrow\|x-y\| \leq \phi(x, y)-\phi(T x, T y) \\
& \Rightarrow\|x-y\| \leq \phi(x, y)-\phi(x, y) \quad[\therefore y \in T x \text { and } z \in T y] \\
& \Rightarrow x-y=0 \\
& \Rightarrow x=y
\end{aligned}
$$

Hence $T$ has a unique fixed point.

\section{Theorem-9}

Let $(X,\|\|$,$) be a complete normed space, and let T: X \rightarrow C B(X)$ be a multi-valued functions such that

$$
H(T x, T y) \leq|\eta|\|x-y\|-|\theta|\|x-y\|, \forall x, y \in X
$$

where $\theta:(0, \infty) \rightarrow(0, \infty)$ is an upper semi-continuous map such that $\forall t \in(0,+\infty)$ and $\frac{\theta(t)}{t}$ is a non-increasing. Then $T$ has a fixed point.

\section{Proof:}

Let $x \in X$ and $y \in T x$. If $y=x$, then $T$ has a fixed and the proof is complete.

Let $\eta(t)=t-\theta(t)$, for each $t>0$. Then $\eta(t)<t$, for each $t>0$ and $\frac{\eta(t)}{t}=1-\frac{\theta(t)}{t}$ is a non-decreasing. Since

$$
\begin{aligned}
& H(T x, T y) \leq\|x-y\|-\left|\frac{\theta}{t}\right|\|x-y\|<\left(1-\left|\frac{\theta}{t}\right|\right)\|x-y\| \\
& \Rightarrow H(T x, T y)<\left|\frac{\eta(t)}{t}\right|\|x-y\|<\|x-y\|
\end{aligned}
$$

Thus there exists $\varepsilon>0$ such that 


$$
\left|\frac{\eta(t)}{t}\right|\|x-y\|=H(T x, T y)+\varepsilon
$$

So there exists $z \in T y$ such that

$$
\begin{gathered}
\|y-z\| \leq H(T x, T y)+\varepsilon=\left|\frac{\eta(t)}{t}\right|\|x-y\| \quad[\text { Using (17)] } \\
<\|x-y\| \quad[\text { Using (16)] } \\
\Rightarrow\|y-z\|<\|x-y\|
\end{gathered}
$$

Again, we suppose that $y \neq z$, then we have

$$
\begin{aligned}
& \|x-y\|-\left|\frac{\eta(t)}{t}\right|\|x-y\| \leq\|x-y\|-\|y-z\| \\
& \Rightarrow\|x-y\|\left(1-\left|\frac{\eta(t)}{t}\right|\right) \leq\|x-y\|-\|y-z\| \\
& \Rightarrow\|x-y\| \leq \frac{\|x-y\|-\|y-z\|}{1-\left|\frac{\eta(t) \mid}{t}\right|} \\
& \Rightarrow\|x-y\| \leq \frac{\|x-y\|}{1-\left|\frac{\eta(t)}{t}\right|}-\frac{\|x-y\|}{1-\left|\frac{\eta(t)}{t}\right|}
\end{aligned}
$$

[Since $\eta(t)$ is non-increasing function and $\|y-z\|<\|x-y\|$ ].

Define $\phi(x, y)=\frac{\|x-y\|}{1-\left|\frac{\eta(t)}{t}\right|}$ if $x \neq y$, otherwise 0 for all $x, y \in X$.

From Equation (19), then we get,

$$
\begin{aligned}
& \|x-y\| \leq \phi(x, y)-\phi(y, z) \\
& \Rightarrow\|x-y\| \leq \phi(x, y)-\phi(T x, T y) \\
& \Rightarrow\|x-y\| \leq \phi(x, y)-\phi(x, y) \quad[\therefore y \in T x \text { and } z \in T y] \\
& \Rightarrow x-y=0 \\
& \Rightarrow x=y
\end{aligned}
$$

Hence $T$ has a unique fixed point.

\section{Remark}

1) If $\gamma:[0,+\infty) \rightarrow[0,+\infty)$ is sub-additive i.e. $\gamma(x+y) \leq \gamma(x)+\gamma(y)$, for each $x, y \in[0,+\infty)$, then it is a non-decreasing continuous map such that $\gamma^{-1}(\{0\})=\{0\}$.

2) Here we show that many of the known Banach contraction generalizations can be deduced and generalized by Caristi's fixed point theorem in normed space and its consequences. 


\section{Conclusion}

Our aim is to discuss the Caristi's fixed point theorem on normed spaces. We hope that this work will be useful for functional analysis related to normed spaces and fixed point theory. All expected results in this paper will help us to understand better solution of complicated theorem. We give an important application and use the fixed point theory related to different branches of mathematics for the solution of physical problems. In future, we will discuss Caristi's fixed point properties related to physical problems.

\section{Acknowledgements}

I would like to thank my respectable teacher Prof. Dr. Nurul Alam Khan for encouragement and valuable suggestions.

\section{Author Contributions}

Authors have made equal contributions for paper.

\section{Conflicts of Interest}

The authors declare that they have no competing interests.

\section{References}

[1] Ekeland, I. (1974) On the Variational Principle. Journal of Mathematical Analysis and Applications, 47, 324-353. https://doi.org/10.1016/0022-247X(74)90025-0

[2] Ekeland, I. (1979) Nonconvex Minimization Problems. Bulletin of the American Mathematical Society, 1, 443-474. https://doi.org/10.1090/S0273-0979-1979-14595-6

[3] Weston, J.D. (1977) A Characterization of Metric Completeness. Proceedings of the American Mathematical Society, 64, 186-188. https://doi.org/10.2307/2041008

[4] Agarwal, R.P. and Khamsi, M.A. (2011) Extension of Caristi's Fixed Point Theorem to Vector Valued Metric Space. Nonlinear Analysis. Theory, Methods \& Applications, 74, 141-145. https://doi.org/10.1016/j.na.2010.08.025

[5] Khamsi, M.A. (2009) Remarks on Caristi's Fixed Point Theorem. Nonlinear Analysis: Theory, Methods \& Applications, 71, 227-231. https://doi.org/10.1016/j.na.2008.10.042

[6] Khojasteh, F., Karapinar, E. and Khandani, H. (2016) Some Applications of Caristi's Fixed Point Theorem in Metric Spaces. Fixed Point Theory and Applications, 2016, Article No. 16. https://doi.org/10.1186/s13663-016-0501-Z

[7] Caristi, J. (1976) Fixed Point Theorems for Mappings Satisfying Inwardness Conditions. Transactions of the American Mathematical Society, 215, 241-251. https://doi.org/10.1090/S0002-9947-1976-0394329-4

[8] Daffer, P.Z., Kaneko, H. and Li, W. (1996) On a Conjecture of S. Reich. Proceedings of the American Mathematical Society, 124, 3159-3162. https://doi.org/10.1090/S0002-9939-96-03659-3

[9] Jachymski, J.R. (1998) Caristi's Fixed Point Theorem and Selections of Set-Valued Contractions. Journal of Mathematical Analysis and Applications, 227, 55-67. https://doi.org/10.1006/jmaa.1998.6074

[10] Reich, S. (1983) Some Problem and Results in Fixed Point Theory. Contemporary 
Mathematics, 21, 179-187.

[11] Mizoguchi, N. and Takahashi, W. (1989) Fixed Point Theorems for Multivalued Mappings on Complete Metric Spaces. Journal of Mathematical Analysis and Applications, 141, 177-188. https://doi.org/10.1016/0022-247X(89)90214-X

[12] Amini-Harandi, A. (2010) Some Generalizations of Caristi's Fixed Point Theorem with Applications to the Fixed Point Theory of Weakly Contractive Set-Valued Maps and the Minimization Problem. Nonlinear Analysis. Theory, Methods \& Applications, 72, 4661-4665. https://doi.org/10.1016/j.na.2010.02.045

[13] Rudin, W. (1991) Functional Analysis. Second Edition, International Editions.

[14] Banach, S. (1922) Sur les opérations dans les ensembles abstraits et leur applicationaux équations intégrales. Fundamenta Mathematicae, 3, 133-181.

https://doi.org/10.4064/fm-3-1-133-181 